\title{
SALIENCIA ABERRANTE Y COMPONENTES DEL MODELO DE GRAY EN EL DESARROLLO DE LA SINTOMATOLOGÍA POSITIVA
}

\section{ABERRANT SALIENCE AND COMPONENTS \\ OF THE GRAY'S MODEL IN THE DEVELOPMENT OF POSITIVE SYMPTOMATOLOGY}

\author{
MARTA SÁNCHEZ-ARJONA CASQUETE DE PRADO ${ }^{1}$, \\ RAFAEl MARTÍNEZ CERVANTES ${ }^{1}$, CRISTINA SENÍN-CALDERÓN ${ }^{2}$ \\ Y JUAN FRANCISCO RODRÍGUEZ-TESTAL ${ }^{1}$
}

Cómo referenciar este artículo/How to reference this article:

Sánchez-Arjona Casquete de Prado, M., Martínez Cervantes, R., Senín-Calderón, C. y Rodríguez Testal, J. F. (2019). Saliencia Aberrante y Componentes del Modelo de Gray en el Desarrollo de la Sintomatología Positiva [Aberrant Salience and Components of the Gray's Model in the Development of Positive Symptomatology]. Acción Psicológica, 16(1), 75-90. https://doi.org/10.5944/ap.16.1.22205

\section{Resumen}

Recientemente, las investigaciones centradas en estudio de las bases de la psicosis han puesto el acento en la saliencia aberrante (Kapur, 2003). Asimismo, se ha demostrado que los sistemas emocionales propuestos por Gray (1982), relacionados con la sensibilidad al castigo y la sensibilidad a la recompensa, influyen en el desarrollo de diversas psicopatologías. Apenas se ha investigado sobre el papel de estos sistemas en la sintomatología positiva. El objetivo de este trabajo fue estudiar el papel

Correspondence address [Dirección para correspondencia]: Marta Sánchez-Arjona Casquete de Prado. Departamento de Personalidad, Evaluación y Tratamiento Psicológicos, Universidad de Sevilla, España.

Email: sanchezarjonamarta@gmail.com

ORCID: Marta Sánchez-Arjona Casquete de Prado (http://orcid.org/0000-0002-0792-7832), Rafael Martínez Cervantes (http://orcid.org/0000-0002-4248-861X), Cristina Senín-Calderón (http://orcid.org/0000-0002-9181-8603) y Juan Francisco Rodríguez-Testal (http://orcid.org/0000-0002-9181-8603).

${ }^{1}$ Universidad de Sevilla, España.

${ }^{2}$ Universitat de Cádiz, España.

Recibido: 11 de junio de 2018 .

Aceptado: 25 de septiembre de 2018 . 
mediador de la sensibilidad al castigo y la sensibilidad a la recompensa en la relación entre la saliencia aberrante y la sintomatología positiva, entendida como ideas de referencia, ideas persecutorias y propensión alucinatoria en una muestra compuesta por 259 estudiantes de la Universidad de Sevilla (España). Los resultados mostraron una mediación parcial de la sensibilidad al castigo y la sensibilidad a la recompensa en el modelo de mediación de las ideas de referencia, así como una mediación parcial de la sensibilidad a la recompensa en el modelo de la propensión alucinatoria. No obstante, no se observó mediación ni de la sensibilidad al castigo ni de la sensibilidad a la recompensa en el modelo de las ideas persecutorias. Según estos resultados, parecer ser que la sensibilidad al castigo y la sensibilidad a la recompensa son características básicas de la personalidad que podrían influir en el desarrollo de algunos síntomas positivos, y por ello, de la psicosis.

Palabras clave: Saliencia aberrante; Personalidad; Sensibilidad al castigo; Sensibilidad a la recompensa; Sintomatología positiva; Psicosis.

\begin{abstract}
Recently, research focused on the study of the bases of psychosis has placed the accent on aberrant salience (Kapur, 2003). Likewise, it has been shown that the emotional systems proposed by Gray (1982), related to sensitivity to punishment and sensitivity to reward, influence the development of various psychopathologies. The role of these systems in psychosis has hardly been investigated. The objective of this work was to study the mediating role of sensitivity to punishment and sensitivity to reward in the relationship between aberrant salience and positive symptomatology, understood as ideas of reference, persecutory ideas and hallucinatory propensity in a sample composed of 259 students of the University of Seville (Spain). The results showed a partial mediation of the sensitivity to punishment and the sensitivity to reward in the mediation model of the ideas of reference, as well as a partial mediation of the SR in the model of the hallucinatory propensity. However, no mediation was observed neither of the sensitivity to punishment nor of the
\end{abstract}

sensitivity to reward in the persecutory ideas model. According to these results, it seems that the sensitivity to punishment and sensitivity to reward are basic characteristics of the personality that could influence the development of some positive symptoms, and therefore, of psychosis.

Keywords: Aberrant salience; Personality; Sensitivity to punishment; Sensitivity to reward; Positive symptomatology; Psychosis.

\section{Introducción}

A lo largo de los años, numerosas investigaciones han ahondado en el estudio de las bases de la psicosis. La psicosis es un fenómeno que se traduce en una distorsión de la realidad, y cuyos indicadores más conocidos y tradicionalmente ligados a ella son la presencia de ideas delirantes (destacando los delirios referenciales y los delirios persecutorios) y las alucinaciones. Van Os, Linscott, MyinGermeys, Delespaul y Krabbendam (2009) sugieren que existe un continuo psicótico, es decir, que en población general se dan, en menor medida, con menor intensidad, o de forma más pasajera, síntomas psicóticos, llamados a menudo experiencias (pseudo)psicóticas. La consideración de un continuo en la psicosis hace dirigir la investigación hacia la población general para estudiar las bases de dichas manifestaciones. Una consecuencia práctica es que, si la psicosis es un fenómeno continuo, se puede prevenir antes de la presencia de la disfunción y malestar estables. No obstante, estas experiencias psicóticas o experimentar síntomas positivos no está directamente relacionado con el desarrollo o la presencia de un trastorno. A lo largo de este estudio, nos centraremos en tres indicadores psicóticos atenuados o sintomatología positiva atenuada, como las Ideas de referencia, Ideas persecutorias y Propensión alucinatoria, ya que se aborda fundamentalmente sobre población no clínica, siendo menos apropiado referir delirios o alucinaciones en sentido estricto.

Las Ideas de Referencia (IRs) son una forma de pensamiento autorreferente, en el cual la propia persona es el foco de su atención. Son comunes en el ser humano, siendo una primera interpretación de los estímulos sociales, es decir, de miradas, gestos, comentarios o acciones 
de otras personas en general. Habitualmente son hechos casuales y neutros que se malinterpretan, y que, aun siendo relevantes para la persona, acaban teniendo un sentido inofensivo (Senín Calderón, Rodríguez-Testal y PeronaGarcelán, 2014). Cuando las IRs son clínicamente significativas consideran delirantes. La definición que realiza el DSM-5 (American Psychiatric Association [APA], 2013) recalca la importancia de distinguir las IR de los delirios de referencia (p. 823). La diferencia se encuentra en la creencia real e incuestionable de la interpretación de los estímulos sociales. Las IRs no son convicciones delirantes, sino ideas relacionadas con la posibilidad de que esos estímulos estén referidos a su persona.

Cicero y Kerns (2011) distinguen dos tipos de pensamiento referencial: un pensamiento agradable (por ejemplo, escuchar una canción y pensar que puede estar escrita para uno/a mismo/a) y un pensamiento desagradable (por ejemplo, pensar que te culparán de un hecho). Estos autores encontraron una fuerte asociación entre el pensamiento referencial desagradable y la paranoia.

La paranoia es la creencia irreal de que otras personas tienen la intención de ocasionarnos un daño. Bebbington et al. (2013) sugieren que es tan común que se puede considerar normal en su sentido estadístico. No obstante, la paranoia también es muy característica de los trastornos psicóticos, siendo los delirios persecutorios muy comunes en estos estados. Estos autores defienden que la paranoia sigue una estructura jerárquica, en la que las ideas persecutorias (en adelante IPs) forman parte de los factores principales para el desarrollo y mantenimiento de los delirios paranoicos. Las IPs tendrían su base en las cogniciones comunes de desconfianza, sensibilidad interpersonal y en las IRs. Las IPs se distinguen de las IRs en que estas últimas no tienen el elemento de la intención de hacer daño (Freeman, 2007). Bell y O'Driscoll (2018) siguen esta misma línea, no obstante, proponen que la paranoia no tendría una estructura jerárquica, sino una estructura en red en la que las IRs serían el factor más central, y en menor medida las IPs. Tomando estas investigaciones en su conjunto, coinciden en que, al igual que las IRs, las IPs son síntomas que se dan tanto en la población clínica como en la no clínica y que forman parte de los componentes de la paranoia.
En cuanto a las alucinaciones, son experiencias sensoriales (auditivas o visuales, por ejemplo), que se dan en ausencia de un estímulo externo (APA, 2013). Este fenómeno se presenta de forma destacada en la esquizofrenia, pero también se pueden presentar en otras psicopatologías, como los trastornos más graves del estado del ánimo, trastornos de personalidad, y otros trastornos psicóticos (Rodríguez-Testal, Perona-Garcelán y Benítez-Hernández, 2011). No obstante, al igual que el resto de síntomas positivos, las alucinaciones las podemos encontrar en la población general sin necesidad de considerarse clínicamente significativo (van Os et al., 2009).

La mayor parte de la literatura acerca de este síntoma se ha centrado en las alucinaciones auditivas verbales (Rodríguez-Testal et al., 2011). Diversos autores defienden que este tipo de alucinaciones ocurren en el momento en el que la persona, tanto población clínica como población no clínica, atribuye erróneamente eventos internos a fuentes externas (Waters, Badcock y Maybery, 2003). En esta misma línea, Perona-Garcelán et al. (2017) defienden que las alucinaciones pueden darse cuando la persona sufre una disociación en el proceso de diálogo interno, es decir, cuando se experimentan las diferentes facetas de uno mismo que componen el diálogo interno como voces de otras personas, es decir, atribuidos a otra persona. Otros autores hablan de diversas disfunciones en los procesos cognitivos, destacando dificultades en la inhibición de información verbal irrelevante (Daalman et al., 2011; Kapur, 2003). Baumeister, Sedgwick, Howes y Peters (2017) exponen que las personas sanas que experimentan alucinaciones auditivas verbales corren más riesgo de padecer algún tipo de psicopatología en el futuro, y muestran un nivel más alto de ideación delirante. No obstante, experimentar estas alucinaciones no es necesariamente un indicador de psicopatología aunque, para su estudio en población general, se alude a la propensión alucinatoria.

En resumen, las IRs, las IPs, y la propensión alucinatoria (en adelante, PA) son síntomas positivos, generalmente considerados atenuados, que pueden encontrarse tanto en la población general como en la población clínica. No obstante, cabría preguntarse cuál es la base de estos síntomas. Recientemente, se ha puesto el acento en el estudio de la saliencia aberrante como un factor central en el 
desarrollo de la psicosis, y por tanto, de los síntomas positivos.

La saliencia aberrante es un proceso mental por el cual la persona experimenta un estado desconcertante y novedoso marcado por la importancia de ciertas percepciones, estados emocionales e ideas. En ciertas situaciones, puede ocurrir que la persona ante determinados estímulos que normalmente han sido valorados como insignificantes, se conviertan en aberrantemente salientes. Una gran variedad de investigaciones han relacionado una disfunción del sistema dopaminérgico con este fenómeno. Uno de los autores que más luz ha arrojado en la comprensión de la saliencia aberrante es Shitij Kapur. Este autor afirma que el sistema dopaminérgico, bajo condiciones normales, es un mediador en la experiencia de detectar la novedad y la recompensa del contexto, lo que hace focalizar nuestra conducta hacia determinados estímulos. Sin embargo, puede ocurrir que una alteración de este sistema provoque una exagerada atención a estímulos insignificantes. Esto quiere decir que la desregulación del sistema dopaminérgico es el creador de la saliencia aberrante estimular (Kapur, 2003). Esta teoría postula que el intento de darle un significado razonable a estas experiencias y/o que las personas atribuyan estímulos internos a fuentes externas puede preceder a la sintomatología positiva (IRs, IPs, y PA).

Las personas que experimentan este proceso mental pueden sentir emociones desde excitación o éxtasis por lo que están viviendo, hasta ansiedad y miedo por los cambios desconocidos, y posiblemente incontrolables que experimentan (Cicero, Kerns y McCarthy, 2010). De esta forma, puede ser que las características temperamentales o elementos más estables de la personalidad influyan en la forma de experimentar fenómenos como la saliencia aberrante y, por tanto, en el desarrollo de la psicosis.

Gray (1982) es uno de los autores más conocidos por sus estudios acerca de la personalidad. Este autor, desarroló la Teoría de la Sensibilidad al Refuerzo, en la cual hace referencia a la existencia de tres sistemas de emoción fundamentales en el ser humano: el Sistema de Aproximación Comportamental (SAC), el Sistema de Huida/Lucha/Inmovilidad (FFFS, siglas en inglés) y el Sistema de Inhibición Comportamental (SIC). El FFFS es el sistema menos estudiado, y se encargaría de las respuesta de huida o lucha ante estímulos aversivos incondicionados. El sistema más estudiado es el SIC, el cual, normalmente funciona como un controlador del comportamiento ante señales de castigo, estímulos novedosos o no reforzantes, y relacionado con la dimensión de ansiedad. El SAC es el responsable de controlar el comportamiento ante estímulos reforzantes, y está relacionado con la dimensión de impulsividad (Torrubia, Ávila, Moltó y Caseras, 2001). Podría decirse que el SAC es el motor que impulsa la actividad y el interés, favorece la búsqueda de recursos, la competitividad por los mismos, y las emociones positivas (Olino, 2016). Si este sistema se relaciona básicamente con la dopamina, el SIC es un sistema de actividad serotoninérgica, y por lo tanto, de inhibición en la agresión, un sistema con capacidad de frenada o, en términos de Gray, un comparador acerca del medio conocido (Gray y McNaughton, 2000), búsqueda de regulación de las emociones negativas, y de obtención de calma y apoyo en los congéneres sistemas opiáceos y oxitocina- (Gilbert, McEwan, Hay, Irons y Cheung, 2007). Aunque se resume diciendo que el SIC actuaría ante situaciones de castigo, y el SAC ante estímulos de recompensa, ambos sistemas intervienen en permanente regulación nombrada como sensibilidad al castigo y a la recompensa.

En esta línea, la sensibilidad al castigo y la sensibilidad a la recompensa, es decir, las características más básicas o temperamentales de la personalidad, podrían influir en la interpretación de los estímulos contextuales. Senín-Calderón, Perona-Garcelán, Fuentes-Márquez y Rodríguez-Testal (2017) demostraron que la sensibilidad al castigo (SC) y la sensibilidad a la recompensa (SR) juegan un papel mediador, junto con otras variables como la depresión y la autoconciencia pública, en la relación entre la ansiedad y las IRs. Esto demuestra que las características temperamentales podrían tener un papel central en el desarrollo de las IRs. Sin embargo, no hay ningún estudio que relacione estas variables (SC y SR) con otros síntomas positivos, como las IPs y la PA, en la población general.

Por tanto, tomando como base el estudio de Senín-Calderón et al. (2017) y, teniendo en cuenta el papel central de la saliencia aberrante en el desarrollo de la sintomatología psicótica, el objetivo de este estudio es averiguar si, al igual que la SC y a la SR juegan un papel mediador entre 
la ansiedad y las IRs, estas dos variables de la personalidad también median en la relación entre la Saliencia Aberrante y la sintomatología positiva (entendida como IRs, IPs, y PA).

Por ello, predecimos que: (a) la Saliencia Aberrante estará relacionada directa y significativamente con la sintomatología positiva: Ideas de Referencia, Ideas Persecutorias y Propensión Alucinatoria; (b) se dará una relación significativa entre la Saliencia Aberrante y la Sensibilidad al Castigo y la Sensibilidad a la Recompensa; (c) existirá una relación significativa entre la Sensibilidad al Castigo y a la Recompensa y la sintomatología positiva: Ideas de Referencia, Ideas Persecutorias y Propensión Alucinatoria), y (d) la Sensibilidad al Castigo y a la Recompensa mediarán en la relación entre la Saliencia Aberrante y la sintomatología positiva: Ideas de Referencias, Ideas Persecutorias y Propensión Alucinatoria.

\section{Método}

\section{Participantes}

La muestra se compuso por 259 sujetos estudiantes universitarios, de los cuales el $79.2 \%$ eran mujeres y el $20.8 \%$ varones. Las edades estaban comprendidas entre 19 y 42 años, con una media de edad de 21.25 años $(D T=2.83)$. El $93.8 \%$ de los participantes eran solteros y el $6.2 \%$ estaban casados o tenían pareja de hecho.

\section{Instrumentos de evaluación}

Evaluación inicial autoadministrada. Identifica las características sociodemográficas de los participantes, la historia clínica del participante, el consumo de drogas y los antecedentes personales y familiares.

Inventario de Saliencia Aberrante (ASI; Cicero et al., 2010). Versión española de Fuentes-Márquez (2015). Este instrumento consta de 29 ítems dicotómicos (Sí/No), los cuales conforman cinco factores que miden la saliencia aberrante y con ella la vulnerabilidad a la psicosis tanto en muestras clínicas como no clínicas. Estos cinco factores son: Significado incrementado, Agudizamiento de los sentidos, Comprensión inminente, Emocionalidad intensificada y Cognición aumentada. Según su autor, este instrumento cuenta con una alta consistencia interna $(\alpha$ de Cronbach de .90). En este estudio, se utilizará la medida global. S halló una consistencia interna, $\alpha$ de Cronbach de .90 .

Green Paranoid Thought Scale (GTPS). Adaptación española por Ibáñez-Casas et al. (2015). Este instrumento está compuesto por 32 ítems, los cuales se puntúan en una escala Likert ordinal de cinco opciones de respuesta desde el 1 ("En absoluto") hasta el 5 ("Totalmente"). Esta escala se divide en dos subescalas compuestas por 16 ítems cada una. La subescala A evalúa las ideas de referencia sociales relevantes en la paranoia, y la subescala B lo relativo a pensamientos persecutorios. Además, ambas escalas permiten medir las dimensiones de convicción, preocupación y estrés. Las puntuaciones oscilan entre 16 y 80 puntos. Según sus creadores, este instrumento cuenta con una adecuada consistencia interna en la subescala A $(\alpha$ de Cronbach: .91) y en la subescala B ( $\alpha$ de Cronbach: .96). En este estudio, la subescala A cuenta con $\alpha$ de Cronbach de .90, así como en la subescala B, $\alpha$ de Cronbach: .94.

Escala de Alucinaciones de Launay-Slade-Revisada (LSHS-R). Adaptación española por Fonseca-Pedrero et al. (2010). Esta escala evalúa la predisposición a experimentar alucinaciones tanto en la población clínica como no clínica. Está compuesto por 12 ítems, los cuales se miden a través de una escala tipo Likert de 4 opciones de respuesta, donde el 1 se corresponde a la respuesta "seguramente no se aplica a ti" y el 4 "seguramente se aplica a ti". Este instrumento diferencia dos tipos de factores que miden la Experiencia alucinatoria y los Eventos mentales vívidos. Se puede obtener de 12 a 48 puntos, indicando una mayor propensión alucinatoria cuanto mayor sea la puntuación obtenida. Según el autor, presenta un adecuado comportamiento psicométrico y consistencia interna ( $\alpha$ de Cronbach: .90). En este estudio, se obtuvo una $\alpha$ de Cronbach de .82 .

The Sensitivity to Punishment and Sensitivity to Reward Questionnaire (SPSRQ). Versión española por Torrubia et al. (2001). Este instrumento está compuesto por 48 ítems con dos opciones de respuesta, los cuales mi- 
den dos escalas formadas cada una por 24 ítems. Una de ellas mide la Sensibilidad a la Recompensa (SR), relacionada con el sistema de activación conductual (conductas dirigidas a la obtención de un refuerzo). La otra escala mide la Sensibilidad al Castigo (SC), la cual está relacionada con el sistema de inhibición conductual (evitación conductual o pasiva en situaciones relacionadas con la posibilidad de obtener una consecuencia negativa). Según el autor, ambas escalas han mostrado buenas propiedades psicométricas: consistencia interna, $\alpha$ de Cronbach de la $\mathrm{SC}, .82$, y $\alpha$ de Cronbach de la SR, de .75. En este estudio, se obtuvieron $\alpha$ de Cronbach de la SC, .85, y $\alpha$ de Cronbach de la SR, .77

\section{Procedimiento}

La muestra fue reclutada mediante muestreo no aleatorio de conveniencia y, en parte de la muestra, se utilizó el procedimiento de bola de nieve a partir de varios colaboradores de la investigación. La muestra se compuso de estudiantes universitarios evaluados entre octubre y enero del 2017. La participación fue voluntaria, siendo los criterios de inclusión: tener más de 18 años y ser españoles nativos. Previamente a la realización de la evaluación, los participantes tuvieron que firmar el consentimiento informado en el que se les explicaba con detalle los objetivos de la investigación, las pruebas utilizadas para la evaluación y la garantía de del anonimato y confidencialidad de los datos.

Se empleó un diseño ex post-facto correlacional predictivo de tipo transversal. No hubo tratamiento de posibles variables extrañas y no se dieron valores perdidos porque se utilizó un formulario electrónico de respuestas forzadas en una única medida. Se excluyeron del estudio los participantes con puntuaciones superiores a tres veces el rango intercuartílico. Los datos se analizaron a través del programa IBM SPSS Statistics 22. Para la descripción de la muestra, se realizaron análisis descriptivos y de frecuencia. Se comprobó el supuesto de normalidad a través de la prueba Kolmogorov-Smirnov para una muestra.

Para las tres primeras hipótesis, se realizó un análisis de correlaciones paramétricas. Para la última hipótesis, se realizaron tres análisis de mediación múltiple, en las que la Saliencia Aberrante actuó como variable independiente, la SC y la SR de variables mediadoras, y las Ideas de Referencia (IRs), Ideas Persecutorias (IPs) y la Propensión Alucinatoria (PA) como variables dependientes en cada caso.

Los análisis de mediación múltiple se llevaron a cabo mediante la macro Process versión 3.0 de Hayes (2013) para SPSS. Los análisis fueron de una cola y la significación estadística se estableció para un intervalo del $95 \%$ y 5000 bootstraps.

\section{Resultados}

En primer lugar, se realizó un análisis descriptivo de las variables a estudiar y se comprobó, a través de la Prueba de Kolmogorov-Smirnov para una muestra, que no se cumplía con el supuesto de normalidad (Tabla 1). Se identificaron 16 casos con puntuaciones superiores a tres veces el rango intercuartílico y se excluyeron del estudio,

Tabla 1

Datos descriptivos de las variables y prueba de Kolmogorov-Smirnov

\begin{tabular}{lrcccrrr}
\hline Variable & $\boldsymbol{N}$ & Mínimo & Máximo & $\boldsymbol{M}$ & $\boldsymbol{D T}$ & $\boldsymbol{K}-\boldsymbol{S}$ & $\boldsymbol{p}$ \\
\hline Saliencia & 259 & 0 & 29 & 8.61 & 5.44 & .96 \\
IRs & 259 & 16 & 58 & 23.61 & 8.21 & .00 \\
IPS & 259 & 16 & 51 & 18.90 & 6.30 & .00 \\
PA & 259 & 12 & 28 & 15.40 & 3.21 & .00 \\
SC & 259 & 0 & 24 & 10.56 & 5.06 & .86 \\
SR & 259 & 0 & 23 & 10.00 & 4.19 & .98 & .00 \\
\hline
\end{tabular}

Nota. IRs: Ideas de Referencia; IPs: Ideas Persecutorias; PA: Propensión Alucinatoria; SC: Sensibilidad al Castigo; SR: Sensibilidad a la Recompensa 
Tabla 2

Correlaciones bivariadas entre las variables del estudio

\begin{tabular}{|c|c|c|c|c|c|c|}
\hline Variable & $M(D T)$ & 1 & 2 & 3 & 4 & 5 \\
\hline 1. Saliencia & $8.26(5.24)$ & - & & & & \\
\hline 2. IRs & $22.32(6.36)$ & $.316^{* *}$ & - & & & \\
\hline 3. IPs & $17.60(3.57)$ & $.312^{* *}$ & $.575^{\star *}$ & - & & \\
\hline 4. PA & $15.11(2.94)$ & $.435^{\star *}$ & $.354^{\star *}$ & $.251^{* *}$ & - & \\
\hline 5. SC & $10.22(4.84)$ & $.287^{* *}$ & $.367^{\star *}$ & $.173^{* *}$ & $.157^{*}$ & - \\
\hline 6. SR & $9.77(4.03)$ & $.229^{* *}$ & $.356^{\star *}$ & .110 & $.351^{\star *}$ & $.165^{* *}$ \\
\hline
\end{tabular}

Nota. IRs: Ideas de referencia; IPs: Ideas Persecutorias; PA: Propensión Alucinatoria; SC: Sensibilidad al Castigo; SR: Sensibilidad a la Recompensa. ${ }^{*} p<.05,{ }^{* *} p<.01$.

resultando una muestra definitiva de 243 sujetos, 52 varones $(21.4 \%)$ y 191 mujeres $(78.6 \%)$ de una edad media de 21.34 años (DT: 2.9).

Para la comprobación de las hipótesis 1, 2 y 3 se llevaron a cabo correlaciones bivariadas entre las variables del estudio (Tabla 2). Se encontraron correlaciones positivas y directas entre la Saliencia Aberrante y la SC y la SR (hipótesis 1), con tamaños de efecto pequeño para la SR $(d=0.47)$ y mediano para la SC $(d=0.59)$. En cuanto a la relación entre la SC y la SR y la sintomatología positiva (hipótesis 2), se encontraron correlaciones positivas y directas entre la SC y las IRs, las IPs y la PA, con tamaños de efecto de pequeño a mediano, respectivamente a los síntomas positivos $d=0.78,0.35$, y 0.31 . Asimismo, se encontraron correlaciones positivas y directas entre la SR y las IRs y la PA, con tamaños de efecto mediano, $d=0.76$ y 0.74 . No obstante, no se halló correlación entre la SR y las IPs $(d=0.22)$. Por último, en relación con la tercera hipótesis, se comprobó que la Saliencia Aberrante correlacionó directa y positivamente con las IRs, las IPs y la
PA, con tamaños de efecto de mediano a grande, respectivamente, $d=0.66,0.65$ y 0.96 .

Se realizaron tres análisis de mediación múltiple para estudiar el papel mediador de las variables SC y SR entre la Saliencia aberrante y la sintomatología positiva (IRs, IPs y PA; hipótesis 4 y objetivo de este estudio). La Tabla 3 , muestra los resultados del análisis de mediación múltiple para la variable dependiente IRs, la Tabla 4 de las IPs, y por último, la Tabla 5 de la PA.

Como podemos observar en los tres análisis, la variable Saliencia aberrante relacionaba significativamente con las variables SC y SR (relación a). Centrándonos en el análisis del modelo de mediación de las IRs (Tabla 3), los resultados mostraron una relación significativa entre las variables mediadoras y la variable IRs, siendo más fuerte la relación que ejerce la SR en ella que la SC (relación b). Asimismo, fue significativa la influencia de la Saliencia aberrante en las IRs (relación total c), disminuyendo, aun siendo todavía significativa, la fuerza de esta relación al incluir las

Tabla 3

Modelo de mediación múltiple con tres variables predictores de las Ideas de Referencia

\begin{tabular}{llllll}
\hline Variables & & $\beta$ & $S E$ & $p$ & $I C 95 \%$ \\
\hline Relación a & Saliencia*SC & .26 & .05 & .00 & $.15-.37$ \\
& Saliencia*SR & .17 & .05 & .00 & $.08-.27$ \\
Relación b & SC*IRs & .35 & .07 & .00 & $.20-.51$ \\
& SR*IRs $^{*}$ & .42 & .09 & .00 & $.24-.60$ \\
Relación total c & Saliencia*IRs & .38 & .07 & .00 & $.23-.53$ \\
Relación directa c' & Saliencia*IRs & .21 & .07 & .00 & $.07-.35$ \\
\hline
\end{tabular}

Nota. IRs: Ideas de referencia; SC: Sensibilidad al Castigo; SR: Sensibilidad a la Recompensa. 
Tabla 4

Modelo de mediación múltiple con dos predictores de las Ideas Persecutorias

\begin{tabular}{llrrrr}
\hline Variables & & $\beta$ & $S E$ & $p$ & $I C 95 \%$ \\
\hline Relación a & Saliencia*SC & .26 & .05 & .00 & $.15-.37$ \\
Relación b & SC*IPs & .06 & .04 & .17 & $-.03-.15$ \\
Relación total c & Saliencia*IPs & .21 & .04 & .00 & $.13-.29$ \\
Relación directa c' & Saliencia*IPs & .19 & .04 & .00 & $.10-.28$ \\
\hline
\end{tabular}

Nota. SC: Sensibilidad al Castigo; IPs: Ideas persecutorias.

variables mediadoras (relación directa c'). Estos resultados en su conjunto mostraron una mediación parcial de la SC y la SR en la relación entre la Saliencia aberrante y las IRs. El test de Sobel mostró que la relación total y directa fueron estadísticamente significativas $(p<.01)$ tanto para $\mathrm{SC}(\mathrm{Z}=3.24)$ como para la SR $(\mathrm{Z}=2.84)$. Esta mediación, según la $R^{2}$ ajustada del Resumen del Modelo, explica el $25.23 \%$ del efecto total.

En el modelo de mediación múltiple de las IPs (Tabla 4), la SR se excluyó previamente del análisis al no correlacionar con la variable dependiente IPs, por lo que la $\mathrm{SC}$ se tomó como la única variable mediadora. Se observó que la SC no se relacionaba con las IPs (relación b). Por ello, aun reduciéndose la fuerza de la relación entre la Saliencia aberrante y las IP al incluir la SC (relación directa c'), no fue válido el modelo por no existir un efecto indirecto de la SC en las IP (relación b).

Por último, se realizó el análisis de mediación múltiple de la PA (Tabla 5) y se comprobó que existía una relación entre SR con la PA, pero no fue de la misma forma con la $\mathrm{SC}$ (relación b). Así mismo, la Saliencia aberrante se relacionó con la PA (relación total c) y, al añadir las variables
SC y SR como mediadoras, se redujo la fuerza de esta relación significativamente (relación directa c'). Estos resultados en su conjunto confirmaron una mediación parcial de la SR en la relación entre la Saliencia Aberrante y las PA. El test de Sobel mostró que la relación total y directa fue estadísticamente significativa $(p<.01)$ para el caso de la SR $(Z=2.81)$. Esta mediación, según la $R^{2}$ ajustada del Resumen del Modelo, explica el $25.56 \%$ del efecto total.

\section{Discusión y conclusiones}

El objetivo general de esta investigación es estudiar el papel mediador de la SC y la SR en la relación entre la Saliencia aberrante y los síntomas positivos: Ideas de referencia (IRs), Ideas persecutorias (IPs), y Propensión alucinatoria (PA).

La primera hipótesis de este estudio plantea que la Saliencia aberrante estaría relacionada directamente y significativamente con la sintomatología positiva: IRs, IPs, y PA. Según los resultados obtenidos, se observó que la sa-

Tabla 5

Modelo de mediación múltiple con tres predictores de la Propensión alucinatoria

\begin{tabular}{llllll}
\hline Variables & & $\boldsymbol{\beta}$ & $\boldsymbol{S E}$ & $\boldsymbol{p}$ & \multicolumn{1}{c}{$\boldsymbol{I C ~ 9 5 \%}$} \\
\hline Relación a & Saliencia*SC & .26 & .05 & .00 & $.15-.37$ \\
& Saliencia*SR & .17 & .05 & .00 & $.08-.27$ \\
Relación b & SC*PA & .00 & .03 & .90 & $-.06-.07$ \\
& SR*PA & .19 & .04 & .00 & $.11-.27$ \\
Relación total c & Saliencia*PA & .24 & .03 & .00 & $.18-.30$ \\
Relación directa c' & Saliencia*PA & .21 & .03 & .00 & $.14-.27$ \\
\hline
\end{tabular}

Nota. SC: Sensibilidad al Castigo; SR: Sensibilidad a la Recompensa; PA: Propensión Alucinatoria. 
liencia aberrante se relacionaba significativamente, y con tamaños de efecto de medios a altos, con la sintomatología positiva (IRs, IPs y PA) por lo que se confirma la hipótesis. Esto coincide con lo planteado por Kapur (2003) acerca del papel central de la saliencia aberrante en el desarrollo de la sintomatología positiva. Por tanto, con base en investigaciones anteriores y al presente estudio, se podría predecir que a mayor experiencia de Saliencia aberrante, mayor es la probabilidad de experimentar sintomatología positiva. No obstante, debemos tener presente que pueden existir otros factores que, al igual que la Saliencia aberrante o en interacción con ella, puedan ser origen de la sintomatología positiva, como la claridad del autoconcepto o la identidad étnica (Cicero y Cohn, 2018). Estos factores no se han considerado en el presente estudio, lo que sugiere que estamos ante un fenómeno complejo y los resultados sólo pueden entenderse de forma tentativa.

La segunda hipótesis (la Saliencia aberrante se relacionará directa y positivamente con la SC y la SR) también fue confirmada. Se encontraron correlaciones positivas y directas entre la saliencia aberrante y la SC y la SR, si bien los tamaños de efecto son aquí más modestos. Gray (1982), postuló la existencia de dos sistemas reguladores de emociones que estarían relacionados con la SC y la SR: el SAC (Sistema de Aproximación Comportamental, relacionado con la SR y emociones placenteras) y el SIC (Sistema de Inhibición Comportamental; relacionado con la $\mathrm{SC}$ y la emoción de ansiedad). Tomando de base esta teoría y los resultados del presente estudio, se podría sugerir que las emociones generadas tras el proceso mental de saliencia aberrante, y por tanto, la forma de experimentar este fenómeno, podrían depender de una mayor o menor sensibilidad al castigo y a la recompensa.

La tercera hipótesis hacía referencia a la existencia de una relación significativa entre la SC y la SR y la sintomatología positiva (IRs, IPs y PA). Esta hipótesis no pudo ser confirmada al completo.

Los resultados mostraron una relación positiva y directa entre la SC y SR y las IRs, con tamaños de efecto mediano, lo que respaldaría lo encontrado por Senín-Calderón et al. (2017) sobre estas variables en su investigación. Tomando en consideración los resultados del estudio de estos autores y los de esta muestra, se podría pronosti- car que una elevada activación de los sistemas de inhibición y activación comportamental pueden hacer aumentar las IRs. Es importante la relación entre la SC y las IRs, ya que una alta SC hace aumentar los estados de alarma ante amenazas, generando altos niveles de ansiedad y de IRs, lo que hace predisponer a la persona a una psicopatología, entrando en riesgo de padecer psicosis (Freeman, 2007). La relación entre la SR y a las IRs puede explicar que la persona tienda a buscar el reforzamiento en los estímulos sociales, fijándose más en este contexto y atribuyéndose a su persona lo que pasa a su alrededor (Senín-Calderón et al., 2017). En este estudio, fue más fuerte la relación entre la SC y las IRs, siendo mínima la diferencia entre ésta y la relación entre la SR y las IRs. Tendría sentido ya que las ideas de referencia suelen estar asociadas a estados emocionales negativos (Senín-Calderón et al., 2014).

Sobre la relación entre la SC y SR y las IPs, se encontró una correlación directa y positiva con la SC, pero no con la SR. Esto puede ser explicado tomando en consideración la idea de que las IPs son uno de los factores principales en el desarrollo y mantenimientos de los delirios paranoides, por lo que los componentes emocionales relacionados con la ansiedad y la preocupación influyen en estas ideas (Bebbington et al., 2013; Bell y O'Driscoll, 2018). Por tanto, una elevada SC puede provocar un estado constante de alarma y ansiedad ante señales de amenazas y llevar a un aumento de las IPs. No obstante, la fuerza de la relación en la SC y las IPs es bastante baja, por lo que habría que dejar en duda si realmente es significativa o, más bien, se debe a un efecto no controlado.

Asimismo, se pudo comprobar que existía una relación directa y significativa entre la SC y SR y la PA, dándose un tamaño de efecto grande para la SR. Esta relación sugiere que una elevada SR hace que se tienda a buscar reforzadores en el contexto, por lo que puede ocurrir que las personas que normalmente son propensas a las alucinaciones experimenten más sensaciones de este tipo (por ejemplo, escuchar un estímulo interno, como un pensamiento, y atribuirlo erróneamente a la voz de otra persona). Esto es importante ya que pueden correr más riesgo de padecer algún tipo de psicopatología en el futuro y mostrar mayor ideación delirante (Baumeister et al., 2017). La relación con la SC es bastante débil por lo que no se descarta que 
la significación se deba, al igual que la relación entre la $\mathrm{SC}$ y las IPS, a otros factores no controlados.

Para el estudio de la última hipótesis y objetivo del estudio, se analizó si la SC y la SR mediaban en la relación entre la Saliencia aberrante y la sintomatología positiva (IRs, IPs y PA). En el modelo de mediación entre la Saliencia aberrante y las IRs se pudo confirmar la hipótesis. Se encontró que la SC y la SR mediaban parcialmente en esta relación. Esto sugiere que, si la persona experimenta una desregulación en el sistema dopaminérgico que hace que los estímulos ambientales se conviertan en aberrantemente salientes para la persona y, que además presente una elevada actividad del SAC o del SIC, pueden aumentar las IRs y, por tanto, el riesgo de sufrir algún tipo de psicopatología en el futuro. No obstante, la mediación es parcial, por lo que la SC y la SR no serían suficientes para explicar el efecto total del modelo de mediación. Diversos autores defienden la influencia de otros factores, los cuales no han sido tenidos en cuenta en este estudio, en el desarrollo o mantenimiento de las IRs, como pueden ser la depresión, la ansiedad, la conciencia pública de uno mismo, o la autoestima, entre otros (Cicero y Kerns, 2011; Senín-Calderón et al., 2017).

En relación a las IPs, no se encontró mediación de la $\mathrm{SC}$ en la relación entre la Saliencia aberrante y aquellas. Según los resultados de esta muestra, cuando se experimenta el fenómeno de Saliencia aberrante, la SC no influiría en el aumento de las IPs. Al inicio de este estudio, se hipotetizó que la SC podría mediar en esta relación ya que puede ocurrir que, debido a una elevada actividad del SIC, estímulos sociales aberrantemente salientes para la persona pudiesen ser interpretados como amenazantes, atribuyéndoles intencionalidad por la ansiedad o preocupación de recibir un castigo. Esta hipótesis no se ha confirmado, lo que es desconcertante ya que no encajaría con la literatura existente acerca del papel destacado de la ansiedad y la preocupación ante una amenazada en el pensamiento paranoide (Freeman, 2007; Freeman, Pugh, Vorontsova, Antley y Slater, 2010).

Una posible explicación de estos resultados la podríamos encontrar en la ausencia de control de ciertas variables extrañas. Este tipo de investigaciones implican el estudio de fenómenos muy complejos, por lo que no se des- carta la presencia de algún factor o conjunto de variables desconocidos que esté afectando a los resultados. Asimismo, para controlar el incumplimiento de la normalidad de la muestra, se excluyeron del estudio aquellos sujetos que presentaban puntuaciones extremas, lo que podría estar sesgando la interpretación de los resultados. Las IPs son un fenómeno común pero quizá inestable en población general, por lo que es esperable que no se cumpla el supuesto de normalidad en variables consideradas "clínicas". No obstante, es igualmente posible que haya influido la deseabilidad social, o factores no controlados que permitan que la presencia de indicadores paranoides se contrarresten y resulte más complicada su autoobservación o reconocimiento (Senín-Calderón et al., 2014).

En referencia al modelo de la $\mathrm{PA}$, los resultados mostraron una mediación parcial de la SR en la relación entre la Saliencia aberrante y la PA. Estos resultados pronostican un aumento de la PA si, al darse la Saliencia aberrante, la persona tiende a orientar su comportamiento hacia la recompensa. No obstante, al igual que en el modelo de las IRs, la mediación fue parcial, por lo que la SR explicaría solo una parte del efecto total del modelo. Esto sugiere la posible existencia de otras variables que estén influyendo en la relación entre la Saliencia aberrante y la PA. El papel de la SR como única variable mediadora en el presente modelo tiene sentido ya que el tipo de alucinaciones que experimenta normalmente la población general, concretamente las alucinaciones auditivas verbales, no suelen presentarse como desagradables o negativas, más bien son vividas como un fenómeno curioso. En cualquier caso, encaja la relación entre la Saliencia aberrante y la PA mediada por la SR en la tesis dopaminérgica (Jensen et al., 2007). Estos resultados son relevantes ya que, de constatarse estas relaciones, estas personas correrían un riesgo más alto de padecer algún tipo de psicopatología en el futuro (Baumeister et al., 2017).

El presente trabajo cuenta con una serie de limitaciones, así como puntos fuertes, que han de tenerse en cuenta. En primer lugar, atendiendo a la validez externa la validez externa, los participantes fueron seleccionados por muestreo de conveniencia, lo que sugiere prudencia en la generalización de los resultados. No obstante, el tamaño de la muestra es amplia, por lo que añade valor a los datos 
obtenidos. Asimismo, la mayoría de los participantes eras mujeres, por lo que en futuros estudios sería conveniente asegurar la homogeneidad de la muestra en este sentido. En segundo lugar, la consistencia interna de la escala utilizada para medir la SR, aun siendo aceptable, se puede considerar baja, lo que podría derivar en problemas de error de medida. Sin embargo, el resto de escalas con niveles de consistencia interna adecuados como para asegurar fiabilidad en la medida. En esta línea, es necesario destacar la posible influencia de la deseabilidad social en este estudio, ya que ciertas preguntas relacionadas con la Saliencia aberrante, las IRs, las IPs y la PA, pueden ser interpretadas negativamente debido al estigma social de ciertas percepciones que se relacionan con la locura. Por último, en este estudio se realizaron pruebas paramétricas, las cuales son consideradas bastante robustas para garantizar la validez interna. No obstante, hay que añadir que no se cumplió con el supuesto de normalidad. Así mismo, se ha de tener en consideración la falta de control de otras variables extrañas que pudieran estar influyendo en los resultados.

Como conclusiones, estos resultados nos llevan a sugerir que ciertas características temperamentales, como la SC y la SR, se encuentran aparentemente asociadas al desarrollo o mantenimiento de determinados síntomas positivos como las IRs y la PA. Estos resultados se pueden considerar de interés ya que arrojan luz al entendimiento de la posible influencia de las características más básicas de la personalidad en el desarrollo de la psicosis. Sin embargo, ha de tenerse en cuenta que el diseño transversal y las características correlacionales utilizadas para la medición en este trabajo, implican que no puedan establecerse conclusiones ni causales en la relación entre las variables, ni definitivas desde una perspectiva longitudinal o prospectiva. Sólo han de ser considerados estos resultados de forma orientativa, pudiendo contribuir con un pequeño paso para el objetivo más ambicioso de mejorar la detección temprana y tratamiento de los trastornos del espectro psicótico.

Debido a la estrecha relación de la SC y la SR con las características más básicas de la personalidad, sería conveniente, en futuras investigaciones, la realización de estudios de corte longitudinal con el fin de apreciar el mantenimiento o cambio de las medidas individuales en el tiempo. Así mismo, serían necesarias investigaciones más amplias sobre este fenómeno, en las cuales se pudieran considerar el mayor número posible de factores de riesgo para el desarrollo de la sintomatología positiva, con el fin último de crear un modelo complejo y específico que permitiera predecir, desde la población general, el desarrollo de psicopatologías del espectro psicótico. Una replicación de este modelo en un diseño longitudinal podría representar un conocimiento temprano de los componentes que anuncian la descompensación psicótica, como la saliencia aberrante, asumiendo al mismo tiempo, el riesgo diferenciado para determinadas características temperamentales sobre las que podría intervenirse, aunque fuese de forma igualmente tentativa. Probablemente el mantenimiento de los indicadores positivos de forma atenuada, puede dar una opción de ajuste a las personas con este tipo de indicadores, obteniendo beneficio de una intervención psicológica fundamentada en la reestructuración cognitiva y la aceptación de las experiencias vividas.

\section{Referencias}

American Psychiatric Association (APA) (2013). Diagnostic and Statistical Manual of Mental Disorders, Fifth Edition, DSM-5. Washington, DC: Autor.

Baumeister, D., Sedgwick, O., Howes, O. y Peters, E. (2017). Auditory Verbal Hallucinations and Continuum Models of Psychosis: A Systematic Review of the Healthy Voice-hearer Literature. Clinical Psychology Review, 51, 125-141. https://doi.org/ 10.1016/j.cpr.2016.10.010

Bebbington, P. E., McBride, O., Steel, C., Kuipers, E., Radovanovič, M., Brugha, T., ...Freeman, D. (2013). The Structure of Paranoia in the General Population. British Journal of Psychiatry, 202(6), 419-427. https://doi.org/10.1192/bjp.bp.112.1190 32

Bell, V. y O'Driscoll, C. (2018). The Network Structure of Paranoia in the General Population. Social Psy- 
chiatry and Psychiatric Epidemiology, 53(7), 737744. https://doi.org/10.1007/s00127-018-1487-0

Cicero, D. C. y Cohn, J. R. (2018). The Role of Ethnic Identity, Self-Concept, and Aberrant Salience in Psychotic-Like Experiences. Cultural Diversity and Ethnic Minority Psychology, 24(1), 101-111. https://doi.org/10.1037/cdp0000160

Cicero, D. C. y Kerns, J. G. (2011). Unpleasant and Pleasant Referential Thinking: Relations with Selfprocessing, Paranoia, and other Schizotypal Traits. Journal of Research in Personality, 45(2), 208218. https://doi.org/0.1016/j.jrp.2011.02.002

Cicero, D. C., Kerns, J. G. y McCarthy, D. M. (2010). The Aberrant Salience Inventory: A New Measure of Psychosis Proneness. Psychological Assessment, 22(3), 688-701. https://doi.org/10.1037/a0019913

Daalman, K., van Zandvoort, M., Bootsman, F., Boks, M., Kahn, R. y Sommer, I. (2011). Auditory Verbal Hallucinations and Cognitive Functioning in Healthy Individuals. Schizophrenia Research, 132(2-3), 203-207. https://doi.org/10.1016/j. schres.2011.07.013

Fonseca-Pedrero, E., Lemos-giráldez, S., Paino, M., Sierra-Baigrie, S., Villazón-García, Ú., González, P. G., ...Muniz, J. (2010). Dimensionality of Hallucinatory Predisposition : Confirmatory Factor Analysis of the Launay-Slade Hallucination Scalerevised in college students. Anales De Psicologia, 26(1), 41-48.

Freeman, D. (2007). Suspicious Minds: The Psychology of Persecutory Delusions. Clinical Psychology Review, 27(4), 425-457. https://doi.org/10.1016/ j.cpr.2006.10.004

Freeman, D., Pugh, K., Vorontsova, N., Antley, A. y Slater, M. (2010). Testing the Continuum of Delusional Beliefs: An Experimental Study using Virtual Reality. Journal of Abnormal Psychology, 119(1), 83-92. https://doi.org/10.1037/a0017514
Fuentes-Márquez, M. S. (2015). La saliencia aberrante como base para la sintomatología psicótica [Aberrant Salience as a Basis for Psychotic Symptomatology] (tesis doctoral). Universidad de Sevilla, Sevilla, España.

Gilbert, P., McEwan, K., Hay, J., Irons, C. y Cheung, M. (2007). Social Rank and Attachment in People with a Bipolar Disorder. Clinical Psychology and Psychotherapy, 14(1), 48-53. https://doi.org/ 10.1002/cpp.508

Gray, J.A. (1982). Précis of The Neuropsychology of Anxiety: An Enquiry into the Functions of the Septo-hippocampal System. The Behavioral and Brain Sciences, 5, 469-534. https://doi.org/10. 1017/S0140525X00013066

Gray, J. A. y McNaughton, N. (2000). The Neuropsychology of Anxiety. An Enquiry into the Functions of the Septo-Hippocampal System (2 ed.). New York, NY: Oxford University Press.

Hayes, A. F. (2013). Introduction to mediation, moderation, and conditional process analysis: $A$ regression-based approach. Nueva York, NY: Guilford Press.

Ibáñez-Casas, I., Femia-Marzo, P., Padilla, J. L., Green, C. E. L., De Portugal, E. y Cervilla, J. A. (2015). Adaptación española de las Escalas de Pensamiento Paranoide de Green [Spanish Adaptation of Green Paranoid Thinking Scales]. Psicothema, 27(1), 7481. https://doi.org/10.7334/psicothema2014.103

Jensen, J., Smith, A. J., Willeit, M., Crawley, A. P., Mikulis, D. J., Vitcu, I. y Kapur, S. (2007). Separate Brain Regions Code for Salience vs. Valence during Reward Prediction in Humans. Human Brain Mapping, 28(4), 294-302. https://doi.org/10. 1002/hbm.20274

Kapur, S. (2003). Psychosis as a State of Aberrant Salience: A Framework Linking Biology, Phenomenology, and Pharmacology in Schizophrenia. American Journal of Psychiatry, 
160, 13-23. https://doi.org/10.1176/appi.ajp.160. 1.13

Olino, T. M. (2016). Future Research Directions in the Positive Valence Systems: Measurement, Development, and Implications for Youth Unipolar Depression. Journal of Clinical Child and Adolescent Psychology, 45(5), 681-705. https://doi.org/10.1080/15374416.2015.1118694

Perona-Garcelán, S., Bellido-Zanin, G., Senín-Calderón, C., López-Jiménez, A. M. y Rodríguez-Testal, J. F. (2017). Adaptación al español del Varieties of Inner Speech Questionnaire (VIQS). Estudio de la relación entre diálogo interno, disociación y propensión a las alucinaciones [Adaptation to Spanish of the Varieties of Inner Speech Questionnaire (VIQS). Study of the relationship between internal dialogue, dissociation and propensity to hallucinations]. Clinica Y Salud, 28(2), 93-100. https://doi.org/10.1016/j.clysa. 2017.02.001

Rodríguez-Testal, J. F., Perona-Garcelán, S. y BenítezHernández, M. (2011). Trastornos Psicóticos [Psychotic Disorders]. En J. F. Rodríguez-Testal y P. J. Mesa-Cid (Eds.), Manual de psicopatología clínica (pp. 123-210). Madrid, España: Pirámide.

Senín-Calderón, C., Perona-Garcelán, S., FuentesMárquez, S. y Rodríguez-Testal, J. F. (2017). A Mediation Model for Ideas of Reference: The Role of the Gray Model, Self-Consciousness, and Emotional Symptoms. Psychological Reports, 120(3), 443-459. https://doi.org/10.1177/003329 4117693593
Senín Calderón, C., Rodríguez Testal, J. F. y Perona Garcelán, S. (2014). El pensamiento referencial: aspectos psicopatológicos y del desarrollo [Referential Thinking: Psychopathological and Developmental Aspects]. Leipzig, Germany: Amazon.

Torrubia, R., Ávila, C., Moltó, J. y Caseras, X. (2001). The Sensitivity to Punishment and Sensitivity to Reward Questionnaire (SPSRQ) as a Measure of Gray's Anxiety and Impulsivity Dimensions. Personality and Individual Differences, 31(6), 837-862. https://doi.org/10.1016/S0191-8869(00) 00183-5

Van Os, J., Linscott, R. J., Myin-Germeys, I., Delespaul, P. y Krabbendam, L. (2009). A Systematic Review and Meta-analysis of the Psychosis Continuum: Evidence for a Psychosis Proneness-PersistenceImpairment Model of Psychotic Disorder. Psychological Medicine, 39(2), 179-195. https://doi.org/ 10.1017/S0033291708003814

Waters, F. A. V., Badcock, J. C. y Maybery, M. T. (2003). Revision of the Factor Structure of the Launay-Slade Hallucination Scale (LSHS-R). Personality and Individual Differences, 35(6), 1351-1357. https://doi.org/10.1016/S0191-8869 (02)00354-9 


\title{
ABERRANT SALIENCE AND COMPONENTS OF THE GRAY'S MODEL IN THE DEVELOPMENT OF POSITIVE SYMPTOMATOLOGY
}

\author{
MARTA SÁNCHEZ-ARJONA CASQUETE DE PRADO ${ }^{1}$, \\ RAFAEl MARTÍNEZ CERVANTES ${ }^{1}$, CRISTINA SENÍN-CALDERÓN ${ }^{2}$, \\ AND JUAN FRANCISCO RODRÍGUEZ-TESTAL ${ }^{1}$
}

\section{EXTENDED SUMMARY}

\section{Introduction}

Ideas of reference (IRs), persecutory ideas (PIs) and hallucinatory propensity (HP) are positive symptoms, generally attenuated, which may correspond to both the general population and the clinical population. However, one might ask what the basis of these symptoms is. Recently, research focused on the study of the bases of psychosis has placed the accent on aberrant salience (Kapur, 2003). Aberrant salience is a mental process by which the person experiences a disconcerting and novel state marked by the importance of certain perceptions, emotional states and ideas. In certain situations, it may happen that the person before certain stimuli that have normally been valued as insignificant, become aberrantly outgoing. Therefore, from the approach of Kapur (2003) it is postulated that the attempt to give a reasonable meaning to these experiences and / or that people attribute internal stimuli to external sources may precede the positive symptomatology (IRs, PIs, and HP).

Likewise, it has been shown that the emotional systems proposed by Gray (1982), related to sensitivity to punishment (SP) and sensitivity to reward (SR), influence the development of various psychopathologies. The role of these systems in psychosis has hardly been investigated. The SP and the SR, that is, the most basic or temperamental characteristics of the personality, could influence the interpretation of contextual stimuli. Senín-Calderón, Perona-
Garcelán, Fuentes-Márquez, and Rodríguez-Testal (2017) demonstrated that sensitivity to punishment (SP) and sensitivity to reward (SR) play a mediating role, along with other variables such as depression and public self-awareness, in the relationship between anxiety and IRs. This shows that temperamental characteristics could have a central role in the development of IRs. However, there is no study that relates these variables (SP and SR) with other positive symptoms, such as PIs and HP, in the general population.

The objective of this work was to study the mediating role of SP and SR in the relationship between aberrant salience and positive symptomatology, understood as ideas of reference (IRs), persecutory ideas (PIs) and hallucinatory propensity (HP).

It is predicted that: (a) the Aberrant Salience will be directly and significantly related to the positive symptomatology: Ideas of Reference, Persecutory Ideas and Hallucinatory Propensity; (b) there will be a significant relationship between the Aberrant Salience and the Sensitivity to Punishment and the Sensitivity to the Reward; (c) there will be a significant relationship between the Sensitivity to Punishment and Reward and the positive symptomatology: Ideas of Reference, Persecutory Ideas and Hallucinatory Propensity, and (d) Sensitivity to Punishment and Reward will mediate in the relationship between the Aberrant Salience and the positive symptomatology: Ideas of References, Persecutory Ideas and Hallucinatory Propensity. 


\section{Participants}

\section{Method}

A sample of 259 students of the University of Seville (Spain), of which $79.2 \%$ were women and $20.8 \%$ were men. The ages were between 19 and 42 years old, with an average age of 21.25 years $(\mathrm{SD}=2.83) .93 .8 \%$ of the participants were single and $6.2 \%$ were married or had a common-law partner.

\section{Instruments}

Self-reported tests were administered: The Aberrant Salience Inventory (ASI; Cicero et al., 2010), Spanish version of Fuentes-Márquez (2015); Green Paranoid Thought Scale (GTPS), Spanish adaptation by IbáñezCasas et al. (2015); the Launay-Slade-Revised Hallucination Scale (LSHS-R), Spanish adaptation by FonsecaPedrero et al. (2010), and The Sensitivity to Punishment and Sensitivity to Reward Questionnaire (SPSRQ), Spanish version by Torrubia et al. (2001).

\section{Procedure}

The sample was recruited through non-random sampling of convenience and, in part of the sample, the snowball procedure was used from several research collaborators. The sample was composed of university students evaluated between October and January 2017. The participation was voluntary, and the inclusion criteria were: being over 18 years old and being native Spaniards. Prior to the evaluation, the participants had to sign the informed consent in which they explained in detail the objectives of the research, the tests used for the evaluation and the guarantee of anonymity and confidentiality of the data. An ex post-facto correlational predictive cross-sectional design was used.

\section{Results}

In the verification of hypotheses 1,2 , and 3 positive and direct correlations were found between the Aberrant Salience and the SP and the SR (hypothesis 1), with small effect sizes for the SR $(d=0.47)$ and medium for the SP $(d=0.59)$. Regarding the relationship between CS and SR and positive symptomatology (hypothesis 2), positive and direct correlations were found between CS and IRs, PIs and BP, with small to medium effect sizes $(d=0.78,0.35$, and 0.31 , respectively). Likewise, positive and direct correlations were found between SR and IRs and HP, with medium effect sizes, $d=0.76$ and 0.74 . However, no correlation was found between SR and PIs $(d=0.22)$. Finally, in relation to the third hypothesis, it was found that Aberrant Salience correlated directly and positively with IRs, PIs and HP, with effect sizes from medium to large, $d=0.66,0.65$, and 0.96 , respectively.

Three multiple mediation analyzes were performed to study the mediating role of SP and SR variables between aberrant salience and positive symptomatology (IRs, PIs and HP) (hypothesis 4 and central objective in this study).

The results for the IRs showed a partial mediation of the SP and the SR in the relationship between the Aberrant Salience and the IRs. The Sobel test showed that the total and direct relationship were statistically significant $(p<.01)$ for both SC $(Z=3.24)$ and SR $(Z=2.84)$. This mediation, according to the adjusted $R^{2}$ of the Model Summary, explains $25.23 \%$ of the total effect. In the case of the PIs, it was observed that the SC was not related to the PIs (relationship b). Therefore, even reducing the strength of the relationship between the aberrant salience and PIs to include the SC (direct relationship c '), the model was not valid because there was no indirect effect of the SC in the PIs (relationship b). For HP, the results confirmed a partial mediation of SR in the relationship between Aberrant Salience and HP. The Sobel test showed that the total and direct relationship was statistically significant $(p<.01)$ for the SR case $(Z=2.81)$. This mediation, according to the adjusted $R^{2}$ of the Model Summary, explains $25.56 \%$ of the total effect.

\section{Discussion}

The first hypothesis of this study suggests and confirms that aberrant salience is directly and significantly related to the positive symptomatology: IRs, PIs, and HP. 
The second hypothesis (the aberrant salience will be directly and positively related to the SP and the SR) was also confirmed. Positive and direct correlations were found between the aberrant salience and the SC and the SR, although the effect sizes are more modest here.

The third hypothesis referred to the existence of a significant relationship between the SP and the SR and the positive symptomatology (IRs, PIs and PA). This hypothesis could not be total confirmed.

The results showed a positive and direct relationship between the SP and SR and the IRs, with medium effect sizes, which would support the findings of Senín-Calderón et al. (2017) about these variables in their research. Taking into consideration the results of the study of these authors and those of this sample, it could be predicted that a high activation of the systems of inhibition and behavioral activation can increase the IRs. It is important the relationship between the SP and the IRs, since a high SC increases alarm states against threats, generating high levels of anxiety and IRs, which predisposes the person to a psychopathology, entering at risk of suffering psychosis (Freeman, 2007). The relationship between the SR and the IRs can explain that the person tends to seek reinforcement in social stimuli, focusing more in this context and attributing to their person what happens around them (SenínCalderón et al., 2017). In this study, the relationship between the SP and the IRs was stronger, with a minimal difference between this and the relationship between the SR and the IRs. It would make sense since the ideas of reference are usually associated with negative emotional states (Senín-Calderón et al., 2014).

Regarding the relationship between the SP and SR and the PIs, a direct and positive correlation was found with the SP, but not with the SR. This can be explained taking into account the idea that PIs are one of the main factors in the development and maintenance of paranoid delusions, so the emotional components related to anxiety and worry influence these ideas (Bebbington et al. ., 2013, Bell $\&$ O'Driscoll, 2018). Therefore, a high SP can cause a constant state of alarm and anxiety before signals of threats and lead to an increase of the PIs. However, the strength of the relationship in the SP and the PIs is quite low, so we should leave in doubt whether it is really significant or, rather, is due to an uncontrolled effect.
Likewise, it was possible to verify that there was a direct and significant relationship between SP and SR and HP, giving a large effect size for SR. This relationship suggests that a high SR makes it tend to seek reinforcers in the context, so it may happen that people who are prone to hallucinations experience more sensations of this type (for example, listening to an internal stimulus, such as thought, and wrongly attributed to the voice of another person). This is important since they may be more at risk of suffering from some type of psychopathology in the future and show greater delusional ideation (Baumeister et al., 2017).

As regards the last hypothesis, and objective of the study, it was analyzed whether the SP and the SR mediated in the relationship between the aberrant salience and the positive symptomatology (IRs, PIs and HP). In the model of mediation between the aberrant salience and the IRs, the hypothesis could be confirmed. Regarding the PIs, no mediation of the SP was found in the relationship between the aberrant salience and those. Regarding the HP model, the results showed a partial mediation of SR in the relationship between aberrant salience and HP. These results predict an increase in HP if, when the aberrant salience occurs, the person tends to direct their behavior towards the reward. However, as in the IR model, the mediation was partial, so the SR would explain only a part of the total effect of the model.

As conclusions, these results lead us to suggest that certain temperamental characteristics, such as SP and SR, are apparently associated with the development or maintenance of certain positive symptoms such as IRs and HP. These results can be considered of interest since they shed light on the understanding of the possible influence of the most basic characteristics of the personality in the development of psychosis. However, it must be taken into account that the cross-sectional design and the correlational characteristics used for the measurement in this work imply that no conclusions or causalities can be established in the relationship between the variables, nor definitive from a longitudinal or prospective perspective. These results should only be considered in an illustrative way, being able to contribute with a small step towards the more ambitious goal of improving the early detection and treatment of psychotic spectrum disorders. 